\title{
Massive Stellar X-ray Sources in the Galactic Center
}

\author{
Jon C. Mauerhan \\ Department of Physics and Astronomy, University of California, Los Angeles, CA 90095; \\ mauerhan@astro.ucla.edu \\ Michael P. Muno \\ Space Radiation Laboratory, California Institute of Technology, Pasadena, CA 91104; \\ mmuno@srl.caltech.edu \\ Franz E. Bauer \\ Columbia Astrophysical Laboratory, New York, NY 10027 \\ Mark R. Morris \\ Department of Physics and Astronomy, University of California, Los Angeles, CA 90095
}

\begin{abstract}
We present results of a spectroscopic survey of bright near-infrared counterparts to Xray point sources from a deep Chandra survey of the Galactic nuclear bulge. $K$-band spectroscopy has revealed 13 new Wolf-Rayet and O-supergiant counterparts to Chandra soucres. Although they are systematically softer in X-rays than the general Galactic center source population of accretion powered CVs, their X-ray colors indicate a hard component consistent with emission from thermal plasma with $k T>2 \mathrm{keV}$. Such hard X-ray emission is not ubiquitous among single Wolf-Rayet and O stars but is common among Wolf-Rayet+OB binaries with colliding supersonic winds. Although we regard colliding-wind binary hypothesis as the most likely scenario, it remains possible that several of these objects are wind-accreting neutron stars or black holes in supergiant high-mass $\mathrm{X}$-ray binaries, or extraordinary single stars emitting hard X-rays.
\end{abstract}

\section{Introduction}

The nuclear bulge of the Galaxy has been observed by the Chandra X-ray Observatory on numerous occasions over the last decade, producing a catalog of X-ray points greater in number than anywhere else in the Milky Way by an order of magnitude. Although dominated by cataclysmic variables (CVs) with faint infrared (IR) counterparts $\left(K_{s}>18\right.$ mag; Laycock et al. 2005), IR spectroscopy of bright counterparts to the X-ray population has revealed a growing number of massive stellar X-ray sources near the Galactic center (GC), outside of the well known Arches, Quintuplet and Sgr A* star clusters (Muno et al. 2006, Mikles et al. 2006, Mauerhan et al. 2007, Hyodo et al. 2007). The X-ray emission from these sources has been interpreted as being due to Wolf-Rayet (WR) and O stars in colliding-wind binaries (CWBs), wind-accreting neutron stars or black holes in high-mass X-ray binaries (HMXBs), or extraordinary single WR/O stars. The identification of objects such as these has important implications for the mode and recent history of massive star formation in the GC, the tidal disruption of GC stellar clusters, the evolution of massive binaries, and the role of magnetic fields in controlling accretion at low $\dot{M}$ and channeling the supersonic 
winds from $\mathrm{O}$ stars. Here we present the most recent discoveries of WR/O counterparts to Chandra X-ray sources in the GC.

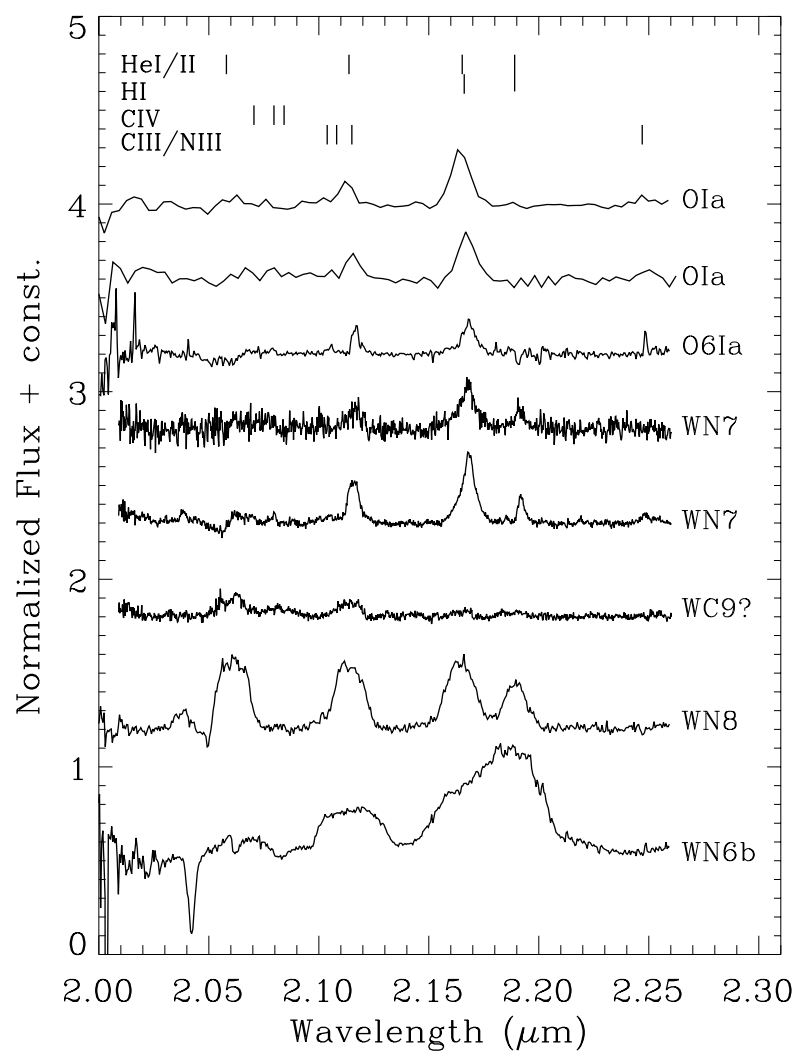

Figure 1. $K$-band spectra of 8 out of the $13 \mathrm{WR} / \mathrm{O}$ stars identified as counterparts to Chandra sources in the Galactic center (obtained at the IRTF, UKIRT, and Keck). The counterparts span the range of post-main-sequence evolution for massive stars, including O-supergiants, and Wolf-Rayet stars of subtype WNE, WNL, and WC.

\section{Observations and Results}

A catalog of over 10,000 X-ray point sources has been assembled from Chandra observations of the inner $2^{\circ} \times 0.8^{\circ}$ of the Galaxy, incorporating all ACIS-I observations of the region 
as of 2007 September (Muno et al. in prep). Assuming a distance of $8 \mathrm{kpc}$ to the GC, the catalog is complete down to $L_{x}>10^{32} \mathrm{erg} \mathrm{s}^{-1}$ over one square degree and an order of magnitude more sensitive in the few arcminutes around Sgr $\mathrm{A}^{*}$, where there is $1 \mathrm{Msec}$ of exposure. We cross-correlated the Chandra GC point-source catalog with NIR catalogs produced by the Simultaneous 3-color Infrared Imager for Unbiased Surveys (SIRIUS) on the 1.4-meter Infrared Science Facility (Nishiyama et al. 2006), and the Two-Micron All-Sky Survey (2MASS; Cutri et al. 2003). In order to select Chandra sources at or beyond the Galactic center, we restict our sample to sources having soft X-ray colors indicative of an interstellar absorbing column of hydrogen with $N_{H}>4 \times 10^{22} \mathrm{~cm}^{-2}$. The IR sample was restricted to sources having colors with $J-H>2.3$ mag and $H-K_{s}>1.1 \mathrm{mag}$, consistent with our adopted cutoff value of $N_{H}$. Further restricting our IR sample to sources with $K_{s}<12$, we produced a list of $125 \mathrm{X}$-ray/IR matches of which $39 \pm 8$ should be genuine, as determined from Monte Carlo simulation.

To determine the nature of these counterparts we obtained $K$-band spectra of 39 stars, of which 13 were discovered to have WR/O counterparts, in excellent agreement with our predicted success rate. This work brings the total number of known X-ray-emitting WR/O stars in the Galactic center to 18, outside the Arches and Quintuplet clusters. For the stars of this work, spectral-types were determined by comparison with spectra of previously identified Galactic WR/O stars in the literature (Hanson et al. 1996, Figer et al. 1996). Figure 1 exhibits $K$-band spectra of 8 of the 13 counterparts discovered, spanning the range of massive star post-main-sequence evolution, from O-supergiants to nitrogen-type WR stars (WN) to carbon-type WR stars (WC). Broad emission lines of $\mathrm{H}$ at $2.16 \mu \mathrm{m}$, and HeI and NIII near $2.12 \mu \mathrm{m}$ dominate the spectra of transitional O supergiants, while HeII emission near $2.19 \mu \mathrm{m}$ emerges in the helium-burning WR stars. The emission lines of the WC9 stars of our sample are extremely diluted, most likely due to continuum emission from dust. The WN6 and WN8 have very broad lines of H, HeI, HeII, and NIII/CIII, indicative of a supersonic wind with $v \approx 2000-3000 \mathrm{~km} \mathrm{~s}^{-1}$. The flat-topped shape of the lines indicates that the wind is extended and optically-thin.

\subsection{X-ray Properties}

Figure 2 shows a plot of hard X-ray color vs. $2-8 \mathrm{keV}$ photon flux. Hard color is defined as $(h-s) /(h+s)$, where $\mathrm{h}$ and $\mathrm{s}$ are the fluxes in the 3.3-4.7 and 4.7-8.0 Chandra bands, respectively. The vertical lines locate the hard color values expected for thermal plasmas of various temperatures, absorbed by $N_{H}=6 \times 10^{22} \mathrm{~cm}^{-2}$. The WR/O counterparts are systematically softer in X-rays than the general Galactic center field population presumed to be accretion powered CVs. However, their X-ray colors do indicate the presence of a hard component with $k T \approx 2-6 \mathrm{keV}$.

\section{Origin of the Hard X-ray Emission}

The hard X-ray emission $(k T>2 \mathrm{keV})$ observed for most of the stars in our sample is not ubiquitous among single WR/O stars. However, X-rays from $\mathrm{WR}+\mathrm{OB}$ binaries with colliding, supersonic stellar winds typically exhibit a hard component. Given the high binary fraction observed among massive stars (Mason et al. 1998), we regard the CWB hypothesis as the most likely. However, we cannot yet rule out the possibility that our sample also contains wind-accreting neutron stars and black holes in HMXBs. However, if there are HMXBs in this sample, their low X-ray luminosities $\left(L_{x} \approx 10^{31-33} \mathrm{erg} \mathrm{s}^{-1}\right)$ compared with known HMXBs would imply a relatively low accretion rate. It has been suggested that the propeller effect produced by a neutron star magnetosphere could prevent the accretion of a 


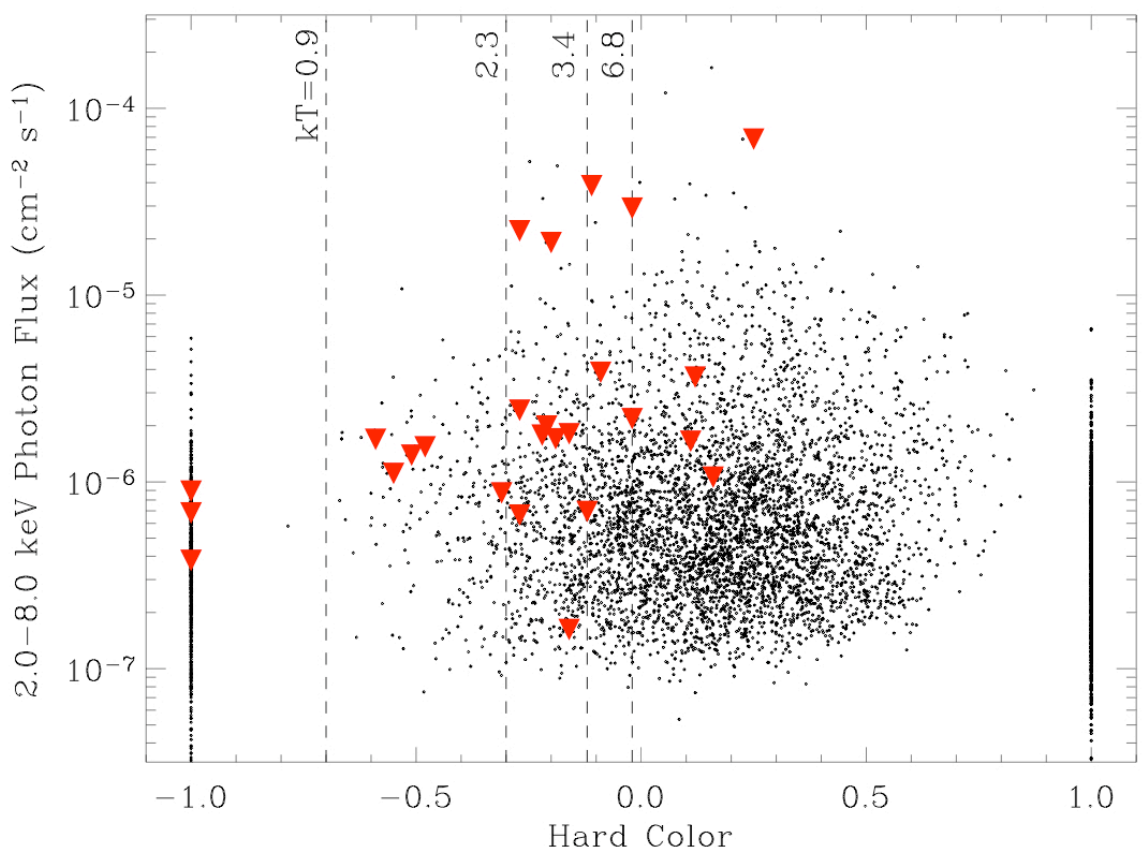

Figure 2. Hard X-ray color vs. flux for the WR/O counterparts to Chandra sources in the Galactic center. In general, the WR/O stars are softer in X-rays than the field population of $\mathrm{CVs}$, but nonetheless exhibit evidence for hard X-ray emission from a $k T>2 \mathrm{keV}$ plasma.

wind at low $\dot{M}$, which would make quiescent HMXBs in the Galactic center undetectable in X-rays (Liu \& Li 2006). Single star scenarios also remain possible. O stars with strong magnetic fields can channel supersonic winds from opposite stellar hemispheres into a selfcollision zone near the stellar equator. Such a model has been developed to explain the hard $\mathrm{X}$-ray emission from the late-O giant $\theta^{1}$ Orionis $\mathrm{C}$ (Wade et al. 2006).

In conclusion, if these WR/O sources are members of CWBs, higher signal-to-noise spectra at medium to high resolution could reveal the spectral signature of a lower luminosity OB companion. Furthermore, multi-epoch spectroscoptic monitoring for velocity variations could also confirm the presence of companions and constrain their masses. These future data will discriminate the various models for hard X-ray emission and elucidate the nature of these objects.

\section{References}

Cutri, R. M. et al. 2003, "Explanatory Supplement to the 2MASS All Sky Data Release" 
Figer, D. F., McLean, I. S., \& Najarro, F. 1997, ApJ, 486, 420

Hanson, M. M., Conti, P. S., \& Rieke, M. J. 1996, ApJS, 107, 281

Hyodo, Y., Tsujimoto, M., Koyama, K., Nishiyama, S., Nagata, T., Sakon, I., Murakami, H., \& Matsumoto, H. 2007, ArXiv e-prints, 712, arXiv:0712.0280

Laycock, S., Grindlay, J., van den Berg, M., Zhao, P., Hong, J., Koenig, X., Schlegel, E. M., \& Persson, S. E. 2005, ApJ, 634, L53

Liu, X. W.\& Li, X. D. 2006, A\&A, 449, L135

Mauerhan, J. C., Muno, M. P., \& Morris, M. 2007, ApJ, 662, 574

Mikles, V. J., Eikenberry, S. S., Muno, M. P., Bandyopadhyay, R. M., \& Patel, S. 2006, ApJ, 651, 408

Muno, M. P., Bower, G. C., Burgasser, A. J., Baganoff, F. K., Morris, M. R., \& Brandt, W. N. 2006, ApJ, 638, 183

Nishiyama, S., et al. 2006, ApJ, 638, 839

Wade, G. A., Fullerton, A. W., Donati, J.-F., Landstreet, J. D., Petit, P., \& Strasser, S. 2006, A\&A, 451, 195 